recreation workers (1.8 SED) had the highest mean exposures. Job title, dosimeter placement, forecast, and hours spent outside were predictors of daily SED. At work, wearing a sleeved shirt (81\% often/always) and hat (73\%) were most prevalent, while seeking shade (12\%) and applying sunscreen (36\%) were least prevalent. Sun protection scores were higher at work than leisure. Hours spent outdoors was a strong determinant for the work and leisure models. Additional leisure model predictors were eye colour, sex, skin type, and job group.

Conclusion Outdoor workers are exposed to high solar UVR levels and use different sun protective behaviours at work and leisure. These findings can inform future monitoring studies and exposure reduction initiatives.

\section{0-296 RADON EXPOSURE AND RISK OF DEATH FROM CIRCULATORY SYSTEM DISEASES AMONG A LARGE COHORT OF URANIUM MINERS - THE PUMA STUDY}

${ }^{1}$ Estelle Rage - de Moissy, David B Richardson, Paul Demers, Minh Do, Nathan DeBono, Nora Fenske, Veronika Deffner, Michaela Kreuzer, Jonathan Samet, Stephen Bertke, Kaitlin Kelly-Reif, Mary Schubauer-Berigan, Ladislav Tomasek, Lydia Zablotska, Charles Wigg. 'Institute for Radiological Protection and Nuclear Safety (IRSN), France

\subsection{6/OEM-2021-EPI. 122}

Introduction Numerous studies highlighted the association between radon exposure and lung cancer risk. Nevertheless the question of radon-related risks for non-cancer diseases, and more specifically circulatory system diseases (CSD) have received limited investigation. Among uranium miners, only one cohort observed an association between radon exposure and CSD.

Objectives To investigate the relationship between cumulative radon exposure and CSD mortality in the PUMA study (Pooled Uranium Miners Analysis), a large international pooled set of cohorts of uranium miners.

Methods The PUMA study includes seven cohorts of uranium miners from Czechia, France, Germany, Canada and USA. Annual radon exposure has been estimated individually and expressed in Working Level Months (WLM). The relationship between cumulative radon exposure, applying a 5-years lag, and CSD death, and more specifically ischemic heart disease and cerebrovascular disease, was assessed with an internal Poisson regression model integrating a linear excess relative risk (ERR) structure, expressed per 100 WLM.

Results The PUMA study includes 119,709 male uranium miners hired between 1942 and 1996. The follow-up duration was between 30-39 years in each of the cohorts, contributing to 4.3 million person-years. The mean value of cumulative radon exposure in individual cohorts ranged between 31 and 580 WLM. At the end of follow-up, 52,450 miners were dead (44\% of the cohort), and among them 17,494 deaths from CSD were recorded. The exposure-risk relationship did not show any increase in CSD risk associated with cumulative radon exposure (ERR/100 WLM $=2.3 .10-4 ; \quad 95 \%$ confidence interval $[-0.0033$; $0.0042])$. No increase in risk was observed for ischemic heart disease or cerebrovascular disease death.

Conclusion The PUMA study has a large study population and a high level of statistical power. These preliminary results did not show any increased risk for CSD mortality among uranium miners.

\section{0-312 OCCUPATIONAL EXPOSURE LEVELS OF SOLAR ULTRAVIOLET RADIATION: AN EPHOR REVIEW}

${ }^{1}$ Else Toft Würtz, Mark Cherrie, Calvin Ge, Kasper Grandahl, Johnni Hansen, Anna Dahlman-Höglund, Ingrid Sivesind Mehlum, Alberto Modenese, Hilde Notö, Cheryl Peters, Jenny Selander, Svetlana Solovieva, Vivi Schlünssen, Marc Wittlich, Henrik Albert Kolstad. 'Aarhus University Hospital, Denmark

\subsection{6/OEM-2021-EPI.123}

Introduction Exposure to solar ultraviolet radiation (UVR) is the main cause of malignant melanoma, squamous cell carcinoma, and basal cell carcinoma of the skin, a risk factor of cataract, and the primary predictor of Vitamin-D levels. The UVR exposure level depends on several factors such as time of day, season, latitude and altitude, activity, and vary across occupations, with high levels reported in various outdoor jobs. Personal measurements are typically reported as full shift cumulative Standard Erythema Dose (SED).

Objective To conduct a systematic review of occupational solar UVR exposure levels.

Methods We performed a PubMed search for literature presenting personal solar UVR exposure levels measured by wearable UVR sensors during work and added papers identified from reference lists. We screened title and abstract of 1,005 papers, full text reviewed 113 papers, and extracted data from 42 included studies. Job descriptions were coded according to the International Standard Classification of Occupations (ISCO-88) on the 4-digit level.

Results In total, UVR exposure levels of 35,345 personal workday measurements of mainly construction workers, farmers, seamen, teachers, expeditioners, and athletes were included. They covered all year, 6am - $6 \mathrm{pm}$ working hours, latitudes from $20-75^{\circ}$ north and $10-68^{\circ}$ south, and altitudes in the $0-8000 \mathrm{~m}$ range. Repeated measurements were included in $76 \%$ of the studies. Eleven different polysulphone film, UV-sensitive spore, or electronic irradiance sensors placed on head, neck, shoulder, chest, back, arm, or wrist were used. Exposure levels varied between 0.4 SED for laboratory workers and 28.6 SED for construction workers.

Conclusion The established measurement database will be used in the development of a general population quantitative job exposure matrix (JEM) as part of the European Exposome Project for Health and Occupational Research (EPHOR). The UV-JEM will be applied in multi-centre European occupational cohort studies of adverse and beneficial effects of exposure to UVR.

\section{Respiratory Effects}

\section{0-39 LIFETIME OCCUPATIONAL EXPOSURES AND CHRONIC OBSTRUCTIVE PULMONARY DISEASE (COPD) RISK IN THE UK BIOBANK COHORT}

${ }^{1}$ Sara De Matteis, Debbie Jarvis, Lucy Darnton, Dario Consonni, Hans Kromhout, Sally Hutchings, Steven Sadhra, David Fishwick, Roel Vermeulen, Lesley Rushton, Paul Cullinan. 'Imperial College London, United Kingdom

\subsection{6/OEM-2021-EPI.124}

Introduction Occupational exposures are important, preventable causes of chronic obstructive pulmonary disease (COPD). We previously found an increased COPD risk among six occupations by analysing lifetime job-histories and lung function data in the population-based UK Biobank cohort. 